\title{
Errata: Iris recognition using Gabor filters optimized by the particle swarm algorithm
}

\author{
Chung-Chih Tsai \\ Jin-Shiuh Taur \\ National Chung Hsing University \\ Department of Electrical Engineering \\ Taiwan \\ Chin-Wang Tao \\ National I-Lan University \\ Department of Electrical Engineering \\ Taiwan
}

This article [J. Electron. Imaging, 18, 023009 (2009)] was originally published online on 15 May 2009 with an error on page 11 , in the first author biography.

The first author's name originally appeared here as "Chen-Chin Tsai." It should read "Chung-Chih Tsai" as per the author affiliations on page 1 .
Also, an acknowledgment was added on page 10 that states "This work is partly supported by the R.O.C. National Science Council through Grant NSC 97-2623-7-005003-D."

All online versions of the article were corrected on 11 June 2009. The article appears correctly in print. 